\title{
Tyrosine phosphatase PTPRD suppresses colon cancer cell migration in coordination with CD44
}

\author{
KOSUKE FUNATO, YUSUKE YAMAZUMI, TAKEAKI ODA and TETSU AKIYAMA \\ Laboratory of Molecular and Genetic Information, Institute of Molecular and Cellular Biosciences, \\ The University of Tokyo, Tokyo 113-0032, Japan
}

Received November 19, 2010; Accepted February 23, 2011

DOI: $10.3892 /$ etm.2011.231

\begin{abstract}
PTPRD is a receptor-type tyrosine-protein phosphatase. Recent analyses of comprehensive mutations and copy numbers have revealed that PTPRD is frequently mutated and homozygously deleted in various types of cancer, including glioblastoma, melanoma, breast and colon cancer. However, the molecular functions of PTPRD in cancer progression have yet to be elucidated. Herein, PTPRD suppressed colon cancer cell migration and was required for appropriate cellcell adhesion. In addition, PTPRD regulated cell migration in cooperation with $\beta$-catenin/TCF signaling and its target CD44. Furthermore, expression levels of PTPRD were down-regulated in highly invasive cancers and were significantly correlated with patient survival. Our findings suggest that PTPRD is required for colon cancer invasion and progression.
\end{abstract}

\section{Introduction}

Receptor-type tyrosine-protein phosphatase $\delta$ (PTPRD) is a receptor type tyrosine-protein phosphatase (RPTP) that is composed of a cell adhesion molecule-like extracellular domain and two cytoplasmic protein tyrosine phosphatase (PTP) domains $(1,2)$. PTPRD is predominantly expressed in the brain and is known to be involved in the guidance and termination of motor neurons during embryonic development (3). PTPRD knockout mice exhibit impaired learning and memory, also indicating that PTPRD is essential for the organization of neural circuits (4).

It has been shown that PTPRD is frequently mutated in various types of cancer, including lung, colon cancer and glioblastoma (5-8). Furthermore, homozygous deletions and epigenetic silencing of $P T P R D$ are also found in these cancers, indicating that PTPRD is a tumor-suppressor gene (9-11).

Correspondence to: Dr Tetsu Akiyama, Laboratory of Molecular and Genetic Information, Institute of Molecular and Cellular Biosciences, The University of Tokyo, 1-1-1 Yayoi, Bunkyo-ku, Tokyo 113-0032, Japan

E-mail: akiyama@iam.u-tokyo.ac.jp

Key words: receptor-type tyrosine-protein phosphatase $\delta$, CD44, cancer cell migration, $\beta$-catenin/TCF signaling
However, the molecular functions of PTPRD in cancer progression are not fully understood.

The extracellular domain of PTPRD was reported to enhance neurite outgrowth in an isoform-specific manner (12). The intracellular domain of PTPRD interacts with cytoskeletal rearrangement factors, such as the Liprin- $\alpha$ family of proteins and MIM (Missing in Metastasis, also known as MTSS1) (13-15). These observations indicate that PTPRD regulates the adhesion and migration of cancer cells and that the loss of PTPRD function promotes cancer progression. In the present study, PTPRD suppressed colon cancer cell migration and was found to be required for appropriate cell-cell adhesion. PTPRD also regulated cell migration in cooperation with $\beta$-catenin/TCF signaling and its target CD44. CD44 is a receptor for hyaluronic acid and other extracellular matrix (ECM) proteins, and is reported to be involved in cancer invasion and metastasis (16). Furthermore, the expression levels of PTPRD were decreased in highly invasive cancers compared to less invasive cases, and were significantly correlated with patient survival. These results implicate PTPRD in colon cancer cell invasion and progression.

\section{Materials and methods}

Cell culture and transfection. DLD-1 cells were cultured in RPMI medium supplemented with $10 \%$ fetal bovine serum (FBS). HEK293T cells were cultured in Dulbecco's modified Eagle's medium (DMEM) containing 10\% FBS. RKO cells were cultured in Eagle's minimum essential medium (MEM) containing $10 \%$ FBS. Plasmids and siRNAs were transfected into cells using Lipofectamine 2000 and RNAiMAX (Invitrogen), respectively.

Plasmid construction. Myc-tagged PTPRD (corresponding to NM_002839.2) was generated by PCR from the cDNA of HEK293T cells and cloned into pcDNA3.1(+) (Invitrogen). For the preparation of GST-fusion proteins, cDNA fragments were subcloned into pGEX-5X (GE Healthcare). GST-fusion proteins were synthesized in Escherichia coli and isolated by adsorption to glutathione-conjugated Sepharose (GSH-Sepharose; Pharmacia). Catalytic-inactive (C1553S) and substrate-trapping (D1521A) mutants of PTPRD were generated using PCR mutagenesis and cloned into pcDNA3.1(+) and pGEX-5X, respectively. 
Antibodies. Mouse monoclonal antibodies to $\beta$-catenin, Plakoglobin and E-cadherin, were obtained from BD Biosciences. The rabbit polyclonal antibody to PTPRD was generated by immunizing rabbits with a GST-fusion protein containing amino acids 1096-1127 of PTPRD. The antibodies were purified by affinity chromatography using columns to which the antigens used for immunization had been linked.

Quantitative RT-PCR. Total RNA was extracted using TRIsure (Bioline) and reverse-transcribed into cDNA using the ReverTra Ace qPCR RT kit (Toyobo). Real-time PCR was performed using LightCycler480 SYBR Green I Master and a LightCycler480 Instrument (Roche). The results were normalized with the detected value for GAPDH. Primers used in RT-PCR were as follows: GAPDH forward, 5'-GCA CCG TCA AGG CTG AGA AC-3'; GAPDH reverse, 5'-TGG TGA AGA CGC CAG TGG A-3'; PTPRD forward, 5'-GCT GCT GCT CCT CAC TTT CT-3'; PTPRD reverse, 5'-CGG GTG TTC GTG TAA ACC TT-3'.

Immunoblotting, immunoprecipitation and GST pull-down assay. Immunoblotting, immunoprecipitation and GST pulldown assays were performed as described previously (17).

Immunofluorescence staining. Cells were fixed with $10 \%$ formalin/PBS for $15 \mathrm{~min}$ and stained overnight with each antibody at $4{ }^{\circ} \mathrm{C}$. Primary antibodies were diluted as follows: anti- $\beta$-catenin (1:500), anti-Plakoglobin (1:500) and antiE-cadherin (1:250). Staining patterns obtained with antibodies were visualized with Alexa Fluor 488-conjugated secondary antibodies (Molecular Probes). Cells were photographed with a Carl Zeiss LSM510 laser scanning microscope (Carl Zeiss).

Migration and scattering assays. Cell migration assays were performed as described previously (18) with minor modifications. Briefly, the underside of the filter membrane was coated with $4 \mu \mathrm{g} / \mathrm{ml}$ type-I collagen (Koken, Japan), $10 \mu \mathrm{g} /$ $\mathrm{ml}$ fibronectin (Sigma-Aldrich) or $10 \mu \mathrm{g} / \mathrm{ml}$ laminin (SigmaAldrich), respectively, and was allowed to air-dry. Transfected DLD-1 cells $\left(1 \times 10^{4}\right.$ cells per well) were added to the upper compartment of the Transwell and allowed to migrate to the underside for $6 \mathrm{~h}$. For RKO cells, $5 \times 10^{3}$ cells were allowed to migrate for $4 \mathrm{~h}$.

For scattering assays, transfected DLD-1 cells $\left(1.2 \times 10^{5}\right)$ were seeded on coverslips (18 $\mathrm{mm}$ in diameter; Matsunami) and cultured for $48 \mathrm{~h}$. Cells were fixed with $10 \%$ formalin/ PBS for $15 \mathrm{~min}$ and stained with TO-PRO 3 (Molecular Probes). For quantification of scattering, staining patterns were photographed and analyzed by Image-J software. The scattering index is defined as the ratio of the area of scattering nuclei divided by that of total nuclei.

RNA interference experiments. Stealth siRNA duplexes against PTPRD were purchased from Invitrogen. The sequences of siRNAs targeting the human PTPRD were: PTPRD-1, 5'-ACA TCA TTC AGT TGT AGC ACA TTT C-3'; PTPRD-3, 5'-ATG ACT TGT ATG TCA CTT GAA AGG G-3'. Validated Stealth negative control siRNA duplex with low GC content (Invitrogen) was used as a control. DNA oligonucleotides encoding shRNA were subcloned into pSuper-retro-puro
(OligoEngine). The sequence of shRNA targeting the human CD44 was 5'-CTG GCG CAG ATC GAT TTG AAT-3'.

Microarray analysis. Microarray datasets and clinical annotations were obtained from the Gene Expression Omnibus (GSE5206 and GSE17537). Signal values of each dataset were log-transformed and normalized before analysis. The probe set IDs were converted to Ensembl gene ID. In cases where one gene ID matched multiple probe set IDs, probe sets with low signal intensity were excluded, and the probe set which shows the most variance across the samples was mapped to the gene ID. Wnt target genes were obtained from The Wnt Homepage (http://www.stanford.edu/ rnusse/wntwindow. html) and converted to Ensembl gene ID. Hierarchical clustering and visualization of the results were performed using GenePattern software, and survival analysis was performed using R software.

\section{Results}

PTPRD suppresses colon cancer cell migration. To investigate the role of PTPRD in cell migration, knockdown experiments were performed using two distinct small interference RNAs (siRNAs). The expression of PTPRD was significantly reduced in DLD-1 cells transfected with the specific siRNAs, but not the control siRNA (Fig. 1A). When siRNA-transfected cells were subjected to migration assays using collagen-coated Boyden chambers, the migratory activity of these cells was significantly increased compared to that of the control siRNAtransfected cells (Fig. 1B). We next performed cell scattering assays and found that the knockdown of PTPRD induced cell scattering without growth factor stimulation (Fig. 1C and D). Consistent with these results, ectopic expression of PTPRD resulted in the inhibition of cell migration (Fig. 1E). Notably, the ectopic expression of a catalytic inactive mutant of PTPRD (PTPRD-CS) also suppressed migration to the same extent as the wild-type, implying that phosphatase activity is dispensable for this suppression.

PTPRD is required for appropriate cell-cell adhesion. It is widely accepted that the disruption of cell-cell adhesion is a crucial step in cancer invasion and metastasis (19). The extracellular domain of PTPRD undergoes homophilic binding, indicating that PTPRD plays a role in cell-cell adhesion (20). We therefore assessed the formation of cell-cell contacts in cells transfected with PTPRD-specific siRNAs by immunostaining for the adhesion molecules, $\beta$-catenin, Plakoglobin and E-cadherin. These adhesion molecules clearly accumulated at the cell-cell contacts in cells transfected with control siRNA, whereas they showed diffused localization in cells transfected with PTPRD-specific siRNAs (Fig. 2A, arrowheads). These results suggest that PTPRD is required for appropriate cell-cell adhesion.

We next investigated whether PTPRD directly interacts with these adhesion molecules. It is known that the membraneproximal D1 domain is catalytically active, while distal D2 domain and substrate-trapping DA mutant are inactive $(1,2)$. We constructed GST-fusion proteins containing one of the two PTP domains (D1 and D2) or a substrate trapping mutant of D1 (DA), respectively. When cell lysates were subjected to 

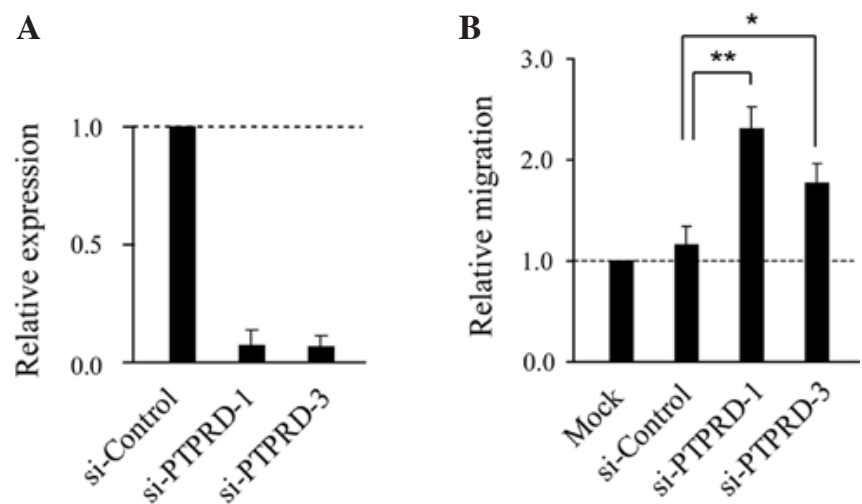

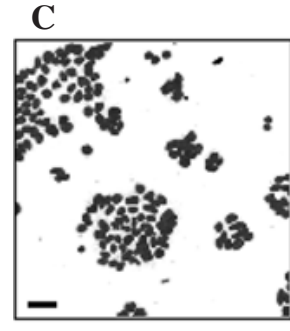

si-Control

D

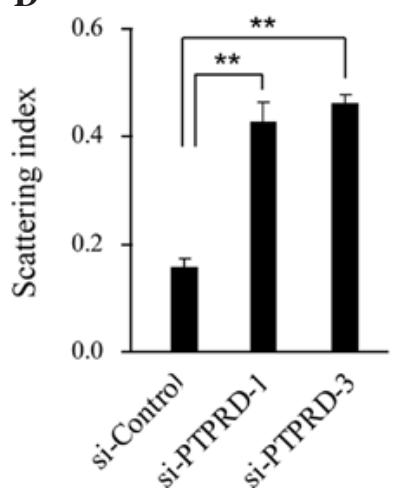

$\mathbf{E}$

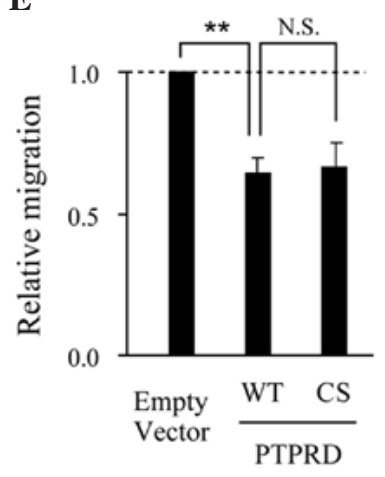

Figure 1. PTPRD suppresses DLD-1 cell migration. (A) Suppression of PTPRD expression by siRNAs. DLD-1 cells were transfected with PTPRDspecific or control siRNA, respectively, and subjected to quantitative real-time RT-PCR. Results shown represent PTPRD mRNA expression relative to GAPDH expression. Error bars represent the mean \pm SD of three experiments in triplicate. (B) Role of PTPRD in the migration of DLD-1 cells. Cells transfected with the indicated siRNAs were added to the upper compartment of the Transwell chamber and allowed to migrate to the underside of the top chamber for $6 \mathrm{~h}$. Error bars represent the mean \pm SEM of at least three independent experiments. (C) Knockdown of PTPRD induced scattering of DLD-1 cells. Cells were transfected with PTPRD-specific or control siRNA, respectively, and nuclear-stained with TO-PRO 3. Scale bars, $50 \mu \mathrm{m}$. (D) Quantification of cell scattering. The scattering index was calculated for the images shown in (C) using Image $J$ software. Bars represent the mean \pm SEM of 10 randomly selected fields. (E) DLD-1 cells were transfected with wild-type (WT) or phosphatase inactive mutant (CS) PTPRD, respectively, and subjected to migration assays. ${ }^{*} \mathrm{p}<0.05,{ }^{* *} \mathrm{p}<0.01$, N.S., not significant $(\mathrm{p}>0.05)$.

pull-down assays with these GST-fusion proteins, $\beta$-catenin was found to interact with D2, but not D1, DA or GST alone (Fig. 2B). To confirm this result, in vivo immunoprecipitation experiments were performed with HEK293T and DLD-1 cells using anti-PTPRD antibody. Both $\beta$-catenin and E-cadherin co-precipitated with PTPRD, whereas N-cadherin did not (Fig. 2C). In these experiments, co-precipitations of $\beta$-catenin

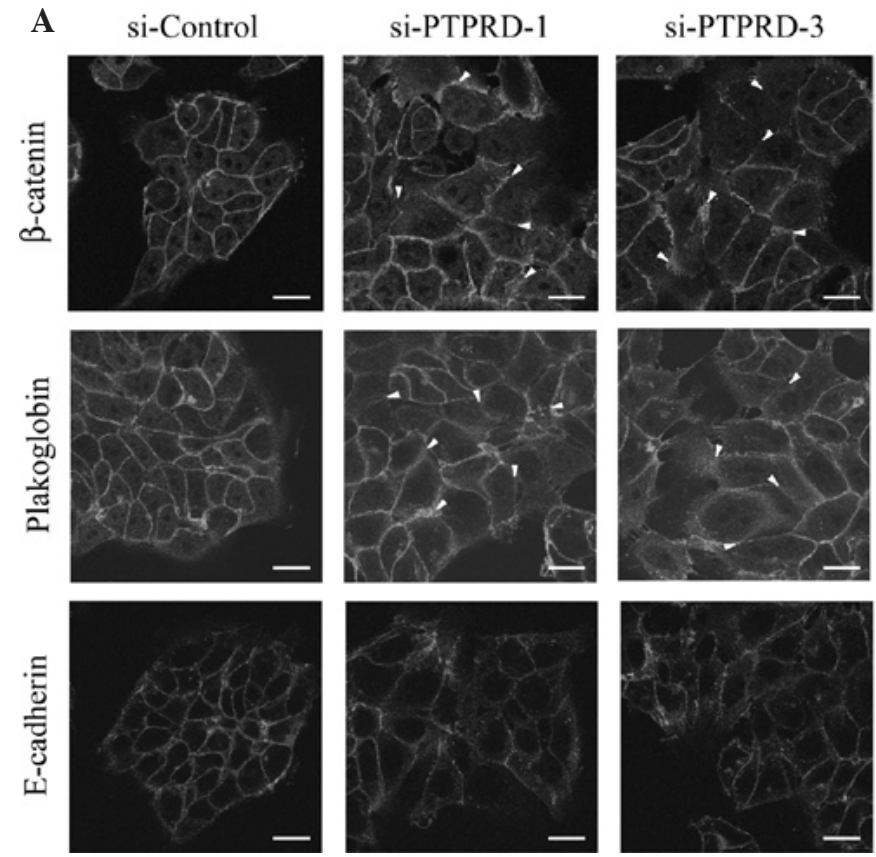

B

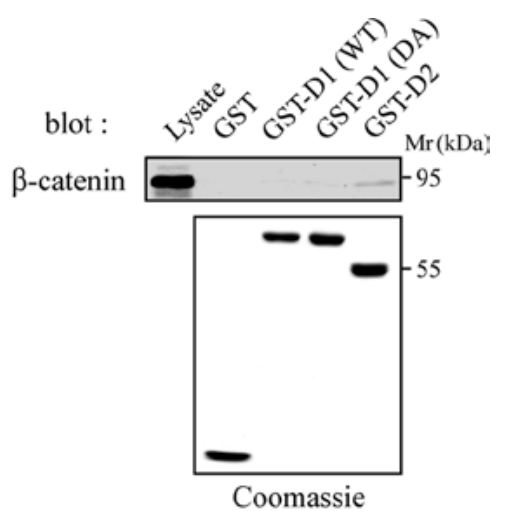

C

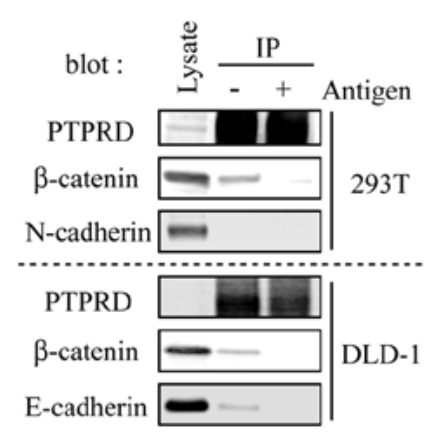

Figure 2. Knockdown of PTPRD weakens cell-cell adhesion. (A) DLD-1 cells were transfected with PTPRD-specific or control siRNA, respectively, and immunostained with the indicated antibodies. Arrowheads indicate diffuse localization of adherent junction proteins. Scale bars, $20 \mu \mathrm{m}$. (B) $\beta$-catenin interacts with the D2 domain of PTPRD. HEK293T cell lysates were subjected to GST pull-down assays using the indicated GST-fusion proteins. WT, wild-type. (C) $\beta$-catenin interacts with PTPRD in vivo. Lysates from HEK293T or DLD-1 cells were immunoprecipitated with an anti-PTPRD antibody. Immunoprecipitates were separated by SDS-PAGE and immunoblotted with the indicated antibodies.

and E-cadherin were inhibited by pre-incubation of the antibody with the antigen used for immunization. These results suggest that PTPRD directly regulates the function of the $\beta$-catenin/E-cadherin complex. 
A

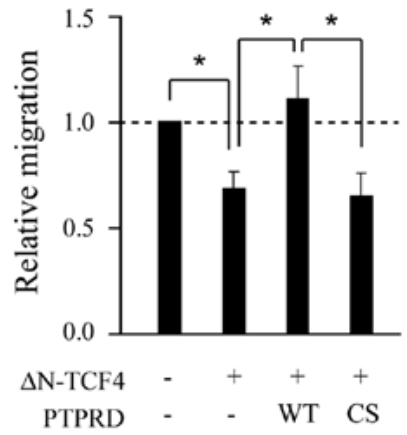

C

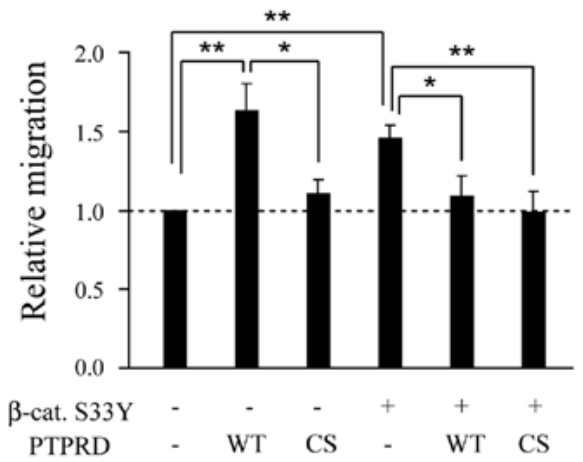

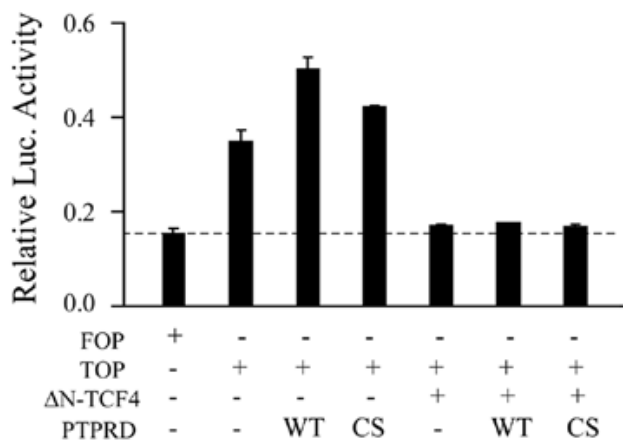

D
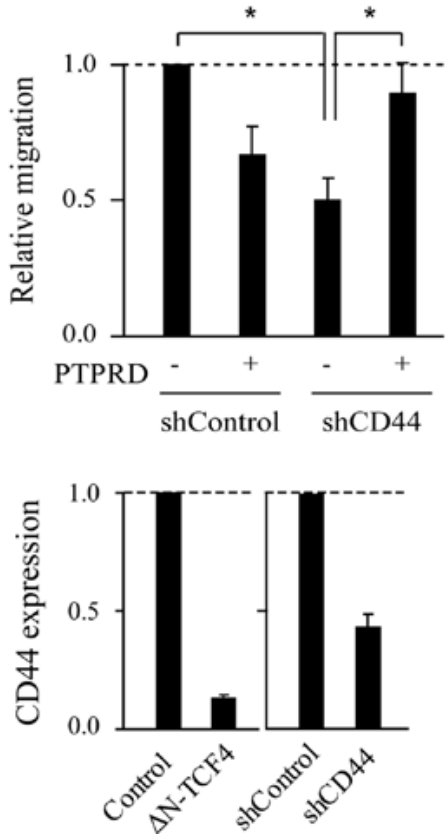

Figure 3. PTPRD-mediated migration and $\beta$-catenin/TCF4 signaling. (A) PTPRD-mediated migration of cells transfected with $\triangle \mathrm{N}-\mathrm{TCF} 4$, a dominantnegative mutant of TCF4. DLD-1 cells were transfected with the indicated plasmids and subjected to migration assays. Bars represent the mean \pm SEM of at least three independent experiments. ${ }^{*} \mathrm{p}<0.05,{ }^{* *} \mathrm{p}<0.01$. (B) DLD-1 cells were transfected with the indicated plasmids. TOP is a reporter plasmid that contains optimal TCF-binding sites upstream of a luciferase gene. FOP contains mutated TCF-binding sites. (C) PTPRD-mediated migration of cells transfected with $\beta$-catenin-S33Y, a constitutively active mutant of $\beta$-catenin. RKO cells were transfected with the indicated plasmids and subjected to migration assays (upper panel) and immunoblotting analysis (lower panel). Bars represent the mean \pm SEM of at least three independent experiments. ${ }^{*} p<0.05,{ }^{* *} p<0.01$. The filled and open arrowheads in the lower panel indicate endogenous and exogenous $\beta$-catenin, respectively. (D) PTPRD-mediated migration of cells transfected with shCD44. Upper panel: DLD-1 cells were transfected with the indicated plasmids or PTPRD and subjected to migration assays. " $\mathrm{p}<0.05$, ${ }^{* *} \mathrm{p}<0.01$. Lower panel: Quantitative RT-PCR analysis was performed to determine the mRNA levels of CD44 in cells transfected with the indicated plasmids.

PTPRD regulates cell migration in cooperation with $\beta$-catenin/TCF signaling. DLD-1 cells harbor biallelic nonsense mutations of APC and express truncated mutant APC. Truncated mutant APC is capable of enhancing cell migration through activation of both $\beta$-catenin/TCF and Rac/Cdc42 signaling (18,21-24). Consistent with previous reports (22-24), ectopic expression of $\triangle \mathrm{N}-\mathrm{TCF} 4$, a dominant-negative form of TCF4, suppressed cell migration (Fig. 3A). The migratory activity of cells transfected with $\triangle \mathrm{N}-\mathrm{TCF} 4$ along with wild-type PTPRD was unexpectedly significantly higher than that of cells transfected with $\triangle \mathrm{N}-\mathrm{TCF} 4$ alone. By contrast, PTPRD-CS, a catalytic inactive mutant, did not show this effect. Thus, it appears that PTPRD stimulates cell migration in a phosphatase activity-dependent manner in the absence of Wnt signaling. To rule out the possibility that PTPRD overcomes or inhibits the dominant-negative effect of $\triangle \mathrm{N}-\mathrm{TCF} 4$, luciferase assays were performed using a TCF-responsive reporter. PTPRD failed to increase its reporter activity when co-expressed with $\triangle \mathrm{N}-\mathrm{TCF} 4$ (Fig. 3B). We next performed migration assays using RKO cells, which contain intact APC and $\beta$-catenin. RKO cells transfected with PTPRD showed increased motility in a phosphatase activity-dependent manner compared to the control cells (Fig. 3C). In contrast, when $\beta$-catenin-S33Y, a constitutively activated form of $\beta$-catenin, was co-expressed, PTPRD suppressed cell migration in a phosphatase activity-independent manner, as observed with DLD-1 cells in Fig. 1E. Taken together, these results suggest that the effect of PTPRD on cell migration depends on the status of $\beta$-catenin/TCF signaling.

We therefore attempted to identify Wnt target genes that are involved in the regulation of PTPRD-dependent migration. Knockdown experiments were performed using shRNAs targeting known Wnt target genes. Similar to cells transfected with PTPRD, cells transfected with a shRNA targeting CD44 (shCD44) showed decreased motility compared to control cells (Fig. 3D). However, cells transfected with PTPRD along with 
A

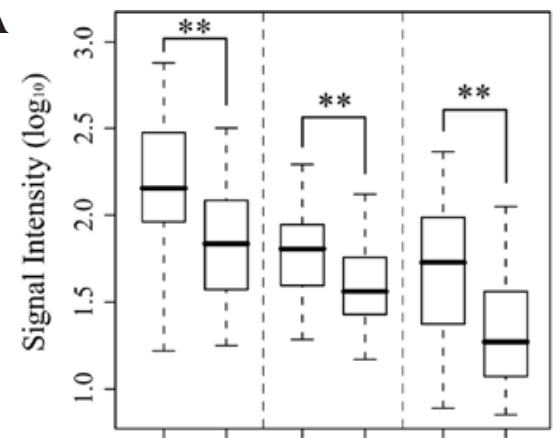

Invasion Low High Low High Low High

Probe $\overline{\text { 214043_at }} \overline{\text { 205712_at }} \overline{213362 \text { at }}$

C

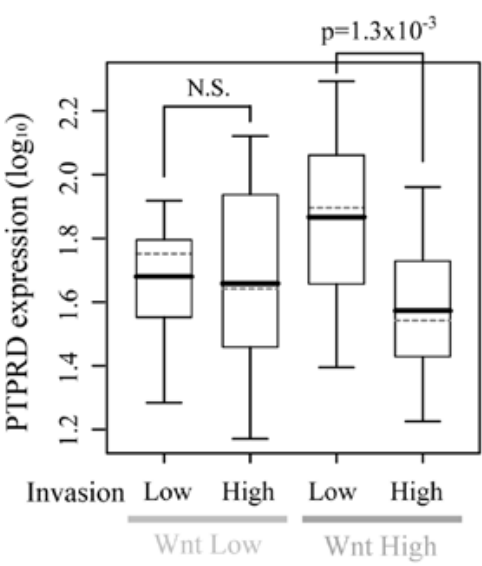

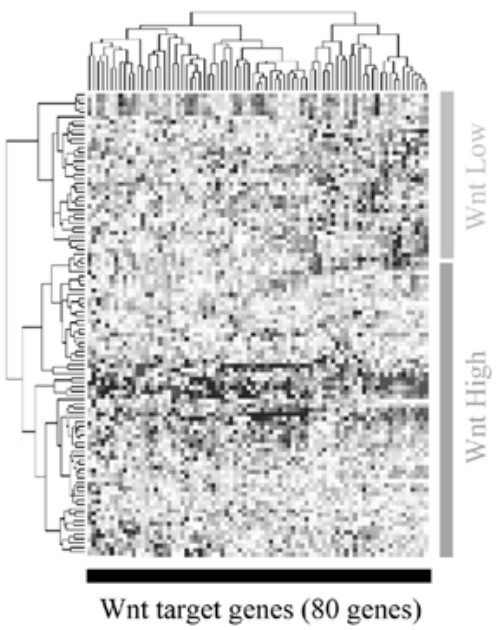

D
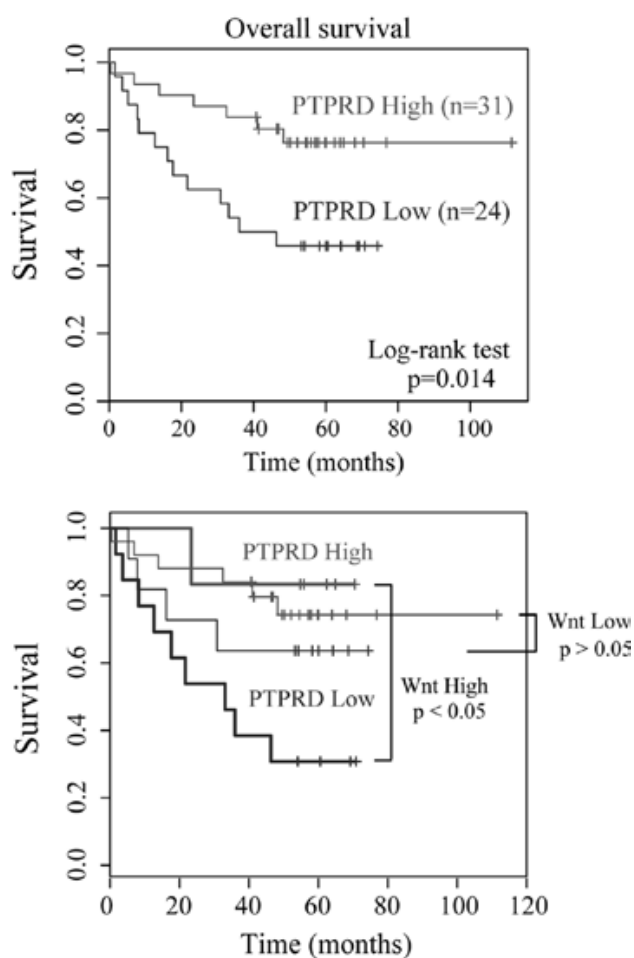

Figure 4. Clinical significance of PTPRD expression in colon cancer patients. (A) PTPRD expression is significantly lower in highly invasive cancers (T3 and T4) than in less invasive cancers. A microarray dataset and clinical annotations were obtained from the Gene Expression Omnibus (GSE5206). (B) Wnt target genes were extracted from the original dataset, and samples were divided into two subgroups using hierarchical clustering. The subgroups were named 'Wnt Low group' and 'Wnt High group', respectively. (C) PTPRD expression was significantly decreased in highly invasive cancers in the Wnt High group, but not in the Wnt Low group. N.S., not significant ( $\mathrm{p}>0.05)$. (D) Expression of PTPRD is significantly correlated with patient survival (Cox's regression: $\mathrm{p}=0.008$ ). A colon cancer prognosis dataset (GSE17537) was analyzed in the whole cohort (upper panel) and separately for the Wnt High group and the Wnt Low group (lower panel).

shCD44 showed increased migratory activity compared to cells transfected with shCD44 alone. These observations suggest that CD44 is involved in PTPRD-dependent migration.

The expression levels of PTPRD are low in highly invasive cancers and correlate with patient survival. To investigate the clinical importance of PTPRD, gene expression profiles of human colon cancers were obtained from a public microarray database. The expression levels of PTPRD were significantly lower in the highly invasive cancers ( $\mathrm{T}$ stage 3 or 4) than in the less invasive cancers (Fig. 4A). There were no significant differences in either lymph node and distal metastasis (data not shown). In most colon cancers, APC or $\beta$-catenin is mutated and $\beta$-catenin/TCF signaling is constitutively activated $(25,26)$. However, the levels of $\beta$-catenin/ TCF signaling differ among cancers according to the type and/or the combinations of mutations (27). We therefore divided the dataset into two subgroups based on the expression levels of known Wnt target genes and termed them the 'Wnt High group' and 'Wnt Low group', respectively (Fig. 4B). Samples from normal colons were classified as the Wnt Low group. An association of $\beta$-catenin/TCF signaling and PTPRD expression was noted in the Wnt High group, but not in the Wnt Low group (Fig. 4C). We also performed Cox's regression analysis and found that both overall survival and disease-free survival were correlated with the expression level 
of PTPRD ( $\mathrm{p}=0.008$ and $\mathrm{p}=0.0025$, respectively; Fig. $4 \mathrm{D}$, upper panel). Furthermore, a significant correlation between PTPRD expression and survival was observed only in the Wnt High group, but not in the Wnt Low group ( $\mathrm{p}=0.036$ and $\mathrm{p}=0.22$, respectively; Fig. $4 \mathrm{D}$, lower panel). Taken together, these results suggest the possibility that the down-regulated expression of PTPRD contributes to the invasive properties and poor patient outcome in human colon cancers in coordination with $\beta$-catenin/TCF signaling.

\section{Discussion}

In the present study, PTPRD suppressed cancer cell migration in cooperation with $\beta$-catenin/TCF signaling. Furthermore, CD44, a target of Wnt signaling $(28,29)$, was required for PTPRD-mediated regulation of cell migration. When $\beta$-catenin/TCF signaling was activated, thereby enhancing CD44 expression, PTPRD suppressed the migratory activity of the cancer cells. It is well known that CD44 interacts with ERM proteins and regulates actin polymerization and cell migration (16). Moreover, aberrant expression and splicing of CD44 were found to be correlated with invasion and patient outcome in colon cancer. Our observation suggests that PTPRD and CD44 regulate cell migration and progression in cooperation. Further study is required to clarify the molecular mechanisms underlying this cooperation. In addition to migration and invasion, CD44 was recently reported to be a marker for cancer stem cells in breast and colon cancer $(30,31)$. Notably, both CD44 and PTPRD were found to be enriched in Lgr5-positive mouse crypt stem cells (32). These observations suggest that PTPRD and CD44 function cooperatively in stem cell maintenance. The role of PTPRD in cancer stem cells is currently under investigation in our laboratory.

We found that reduced expression of PTPRD was correlated with invasive status and poor survival in colon cancer patients. Furthermore, significant correlations were observed only in the patients where Wnt signaling was highly activated. This observation suggests that the effect of PTPRD on cell migration depends on the status of $\beta$-catenin/TCF signaling. Activation of $\beta$-catenin/TCF signaling is also observed in glioblastoma and melanoma $(33,34)$, in which homozygous deletions and epigenetic silencing of PTPRD are found. Hence, investigation of the association of PTPRD and $\beta$-catenin/TCF signaling in these cancers is warranted.

Our migration assays revealed that PTPRD regulates cell migration in a catalytic activity-independent manner. In this regard, it is interesting that PTPRD has been reported to interact with the Liprin- $\alpha$ family of proteins and MIM through its catalytic inactive D2 domain. Liprin- $\alpha$ is a multi-domain scaffolding protein that localizes RPTP to cell focal adhesions and regulates their disassembly. MIM is an actin binding protein that induces cell shape changes, such as the formation of lamellipodia- and filopodia-like structures. The expression level of MIM is negatively correlated with the metastatic activities of various cancer types, indicating its suppressive role in cell migration and invasion. These observations suggest that PTPRD regulates cell migration in conjunction with these cytoskeletal rearrangement factors via its catalytic inactive D2 domain.
We also investigated the role of PTPRD in cell-cell adhesion and showed that PTPRD is required for the formation of appropriate cell-cell contacts. In addition, we found that PTPRD interacts with cell-adhesion molecules, such as $\beta$-catenin and E-cadherin, through its D2 domain. It is known that LAR and PTPRS, homologs of PTPRD, dephosphorylate $\beta$-catenin and thereby stabilize cell adhesion and inhibit cell migration and neurite outgrowth $(35,36)$. It is therefore possible that $\beta$-catenin is a substrate of PTPRD, and that the suppression of PTPRD leads to disruption of the $\beta$-catenin/ E-cadherin complex and cell-cell adhesion.

In the absence of Wnt signaling, PTPRD enhanced cell migration in a phosphatase activity-dependent manner. Some protein tyrosine phosphatases are known to dephosphorylate and activate the Src family of kinases $(37,38)$. Thus, it is also possible that PTPRD activates the Src family of kinases and thereby increases the migratory activity of cancer cells. Furthermore, it has recently been reported that PTPRD dephosphorylates STAT3 and inhibits cell proliferation and transformation (11). In general, tyrosine phosphatases dephosphorylate a wide-range of substrates. Therefore, further efforts to search for novel substrates are required for the better understanding of the function of PTPRD in cancer progression.

\section{Acknowledgements}

The authors thank H. Clevers for providing the FOP/TOP reporter plasmids. This study was supported by Innovative Cell Biology by Innovative Technology, Grants-in-Aid for Scientific Research on Innovative Areas, and in part by the Global COE program (Integrative Life Science Based in the Study of Biosignaling Mechanisms), MEXT, Japan.

\section{References}

1. Tiganis $\mathrm{T}$ and Bennett A: Protein tyrosine phosphatase function: the substrate perspective. Biochem J 402: 1-15, 2007.

2. Ensslen-Craig S and Brady-Kalnay S: Receptor protein tyrosine phosphatases regulate neural development and axon guidance. Dev Biol 275: 12-22, 2004.

3. Stepanek L, Stoker A, Stoeckli E and Bixby J: Receptor tyrosine phosphatases guide vertebrate motor axons during development. J Neurosci 25: 3813-3823, 2005.

4. Uetani N, Kato K, Ogura H, et al: Impaired learning with enhanced hippocampal long-term potentiation in PTPdeltadeficient mice. EMBO J 19: 2775-2785, 2000.

5. Sjöblom T, Jones $\mathrm{S}$, Wood L, et al: The consensus coding sequences of human breast and colorectal cancers. Science 314: 268-274, 2006.

6. Weir B, Woo M, Getz G, et al: Characterizing the cancer genome in lung adenocarcinoma. Nature 450: 893-898, 2007.

7. Network CGAR: Comprehensive genomic characterization defines human glioblastoma genes and core pathways. Nature 455: 1061-1068, 2008

8. Bignell G, Greenman C, Davies H, et al: Signatures of mutation and selection in the cancer genome. Nature 463: 893-898, 2010.

9. Sato M, Takahashi K, Nagayama K, et al: Identification of chromosome arm $9 \mathrm{p}$ as the most frequent target of homozygous deletions in lung cancer. Genes Chromosomes Cancer 44: 405-414, 2005.

10. Solomon D, Kim J, Cronin J, et al: Mutational inactivation of PTPRD in glioblastoma multiforme and malignant melanoma. Cancer Res 68: 10300-10306, 2008.

11. Veeriah S, Brennan C, Meng S, et al: The tyrosine phosphatase PTPRD is a tumor suppressor that is frequently inactivated and mutated in glioblastoma and other human cancers. Proc Natl Acad Sci USA 106: 9435-9440, 2009.

12. Gonzalez-Brito M and Bixby J: Differential activities in adhesion and neurite growth of fibronectin type III repeats in the PTP-delta extracellular domain. Int J Dev Neurosci 24: 425-429, 2006. 
13. Serra-Pagès C, Medley Q, Tang M, Hart A and Streuli M: Liprins, a family of LAR transmembrane protein-tyrosine phosphataseinteracting proteins. J Biol Chem 273: 15611-15620, 1998.

14. Woodings J, Sharp S and Machesky L: MIM-B, a putative metastasis suppressor protein, binds to actin and to protein tyrosine phosphatase delta. Biochem J 371: 463-471, 2003.

15. Gonzalez-Quevedo R, Shoffer M, Horng L and Oro A: Receptor tyrosine phosphatase-dependent cytoskeletal remodeling by the hedgehog-responsive gene MIM/BEG4. J Cell Biol 168: 453-463, 2005.

16. Ponta H, Sherman L and Herrlich P: CD44: from adhesion molecules to signalling regulators. Nat Rev Mol Cell Biol 4: $33-45,2003$.

17. Nakamura T, Hayashi T, Nasu-Nishimura Y, et al: PX-RICS mediates ER-to-Golgi transport of the N-cadherin/beta-catenin complex. Genes Dev 22: 1244-1256, 2008.

18. Kawasaki Y, Sato R and Akiyama T: Mutated APC and Asef are involved in the migration of colorectal tumour cells. Nat Cell Biol 5: 211-215, 2003

19. Jeanes A, Gottardi C and Yap A: Cadherins and cancer: how does cadherin dysfunction promote tumor progression? Oncogene 27: 6920-6929, 2008.

20. Wang $\mathbf{J}$ and Bixby $\mathbf{J}$ : Receptor tyrosine phosphatase-delta is a homophilic, neurite-promoting cell adhesion molecular for CNS neurons. Mol Cell Neurosci 14: 370-384, 1999.

21. Kawasaki Y, Sagara M, Shibata Y, Shirouzu M, Yokoyama S and Akiyama T: Identification and characterization of Asef2, a guanine-nucleotide exchange factor specific for Rac1 and $\mathrm{Cdc} 42$. Oncogene 26: 7620-7267, 2007.

22. Gilles C, Polette M, Mestdagt M, et al: Transactivation of vimentin by beta-catenin in human breast cancer cells. Cancer Res 63: 2658-2664, 2003

23. Gavert N, Conacci-Sorrell M, Gast D, et al: L1, a novel target of beta-catenin signaling, transforms cells and is expressed at the invasive front of colon cancers. J Cell Biol 168: 633-642, 2005.

24. Vignjevic D, Schoumacher M, Gavert N, et al: Fascin, a novel target of beta-catenin-TCF signaling, is expressed at the invasive front of human colon cancer. Cancer Res 67: 6844-6853, 2007.

25. Morin P, Sparks A, Korinek V, et al: Activation of beta-catenin-Tcf signaling in colon cancer by mutations in beta-catenin or APC Science 275: 1787-1790, 1997.
26. Sparks A, Morin P, Vogelstein B and Kinzler K: Mutational analysis of the APC/beta-catenin/Tcf pathway in colorectal cancer. Cancer Res 58: 1130-1134, 1998.

27. Fodde $\mathrm{R}$ and Brabletz $\mathrm{T}$ : Wnt/beta-catenin signaling in cancer stemness and malignant behavior. Curr Opin Cell Biol 19: $150-158,2007$.

28. Wielenga V, Smits R, Korinek V, et al: Expression of CD44 in Apc and Tcf mutant mice implies regulation by the WNT pathway. Am J Pathol 154: 515-523, 1999.

29. Boon E, van der Neut R, van de Wetering M, Clevers H and Pals S: Wnt signaling regulates expression of the receptor tyrosine kinase met in colorectal cancer. Cancer Res 62: 5126-5128, 2002.

30. Al-Hajj M, Wicha M, Benito-Hernandez A, Morrison S and Clarke M: Prospective identification of tumorigenic breast cancer cells. Proc Natl Acad Sci USA 100: 3983-3988, 2003.

31. Du L, Wang H, He L, et al: CD44 is of functional importance for colorectal cancer stem cells. Clin Cancer Res 14: 6751-6760, 2008.

32. Van der Flier L, van Gijn M, Hatzis P, et al: Transcription factor achaete scute-like 2 controls intestinal stem cell fate. Cell 136 903-912, 2009.

33. Zheng H, Ying $\mathrm{H}$, Wiedemeyer R, et al: PLAGL2 regulates Wnt signaling to impede differentiation in neural stem cells and gliomas. Cancer Cell 17: 497-509, 2010.

34. O'Connell $M$ and Weeraratna A: Hear the Wnt Ror: how melanoma cells adjust to changes in Wnt. Pigment Cell Melanoma Res 22: 724-739, 2009.

35. Müller T, Choidas A, Reichmann E and Ullrich A: Phosphorylation and free pool of beta-catenin are regulated by tyrosine kinases and tyrosine phosphatases during epithelial cell migration. J Biol Chem 274: 10173-10183, 1999.

36. Siu R, Fladd C and Rotin D: N-cadherin is an in vivo substrate for protein tyrosine phosphatase sigma (PTPsigma) and participates in PTPsigma-mediated inhibition of axon growth. Mol Cell Biol 27: 208-219, 2007.

37. Harder K, Moller N, Peacock J and Jirik F: Protein-tyrosine phosphatase alpha regulates Src family kinases and alters cellsubstratum adhesion. J Biol Chem 273: 31890-31900, 1998.

38. Zhu S, Bjorge J and Fujita D: PTP1B contributes to the oncogenic properties of colon cancer cells through Src activation. Cancer Res 67: 10129-10137, 2007. 